\title{
Efectos del cambio climático en la distribución del bosque de oyamel
}

\section{Effects Of Climate Change In The Distribution Of Oyamel Forest}

\author{
Gabriel Alexis Araiza-Olivares ${ }^{1}$ \\ Universidad Nacional Autónoma de México, México
}

\begin{abstract}
Resumen
El oyamel (Abies religiosa) es una especie endémica de México; se distribuye en manchones aislados, en las regiones montañosas del centro del país. Para su desarrollo requiere de condiciones medioambientales específicas de temperaturas frescas y alta humedad. Los bosques de oyamel constituyen un gran reservorio de biodiversidad ya que albergan cientos de especies de flora y fauna. Así mismo, cumplen la función de importantes sumideros de carbono y captadores de agua, además de poseer un importante potencial económico puesto que proporcionan materias primas para varias industrias, entre otros servicios ambientales. A pesar de que el cambio climático no es un fenómeno inédito, la velocidad observada en la actualidad hace pensar que su origen no es natural sino antrópico. Este trabajo proyecta los efectos a largo plazo que el cambio climático, de acuerdo al modelo $R E A$, tendría sobre la extensión y distribución de los bosques de oyamel en el Sistema Volcánico Transversal. Los resultados se dividieron en cinco categorías (Muy adecuado, Adecuado, Moderadamente adecuado, Poco adecuado y No adecuado), en función a las condiciones ombrotérmicas de los diferentes forzamientos radiativos del modelo mostraron una sensible disminución en cuanto a la extensión territorial ocupada por este ecosistema, a la vez que se observa un ascenso altitudinal en su distribución.
\end{abstract}

Palabras clave: Oyamel, cambio climático, servicios ambientales, adaptación.

\begin{abstract}
Oyamel (Abies religiosa) is an endemic species of Mexico; the masses that form are distributed in isolated patches, in the mountainous regions of the center of the country. For its development

1 Maestro en geografía, UNAM. Ciudad de México, México. Correo electrónico: g_araiza@ciencias.unam.mx. https://orcid.org/0000-0003-2132-1949
\end{abstract}


requires specific environmental conditions of cool temperatures and high humidity. Oyamel forests are a great reservoir of biodiversity since they house hundreds of species of flora and fauna. Likewise, they fulfill the function of important carbon sinks and water collectors, besides having an important economic potential since they provide raw materials for several industries, among other environmental services. Although climate change is not an unprecedented phenomenon, the speed observed today suggests that its origin is not natural but anthropic. This paper analyzed the longterm effects that climate change, according to the REA model, would have on the extension and distribution of oyamel forests in the Transversal Volcanic System. The results were divided into five categories (Very adequate, Adequate, Moderately adequate, Unsuitable and Unsuitable), depending on the ombrothermic conditions of the different radiative forcings of the model showed a significant decrease in the territorial extent occupied by this ecosystem, while observing an altitudinal rise in its distribution.

Keywords: Oyamel, climate change, environmental services, adapation.

\section{Introducción}

La Tierra absorbe radiación solar y esta es redistribuida a través de la circulación de la atmósfera y el océano de la zona ecuatorial hacia los polos, la energía es irradiada de la superficie al espacio hasta alcanzar el equilibrio entre la radiación absorbida y emitida, sin embargo, la variación de estos factores puede derivar en alteraciones en el clima (Magaña, 2004).

Las emisiones de gases de efecto invernadero derivadas de las actividades humanas han aumentado desde la era preindustrial debido al crecimiento demográfico y económico. De acuerdo con el Panel Intergubernamental de Expertos sobre Cambio Climático, IPCC, por sus siglas en inglés (2014) entre el año 2000 y 2010 las emisiones de gases de efecto invernadero alcanzaron un máximo histórico, las concentraciones de bióxido de carbono $\left(\mathrm{CO}_{2}\right)$, metano $\left(\mathrm{CH}_{4}\right)$ y óxido nitroso (NO) lograron los niveles más altos en 800 mil años, a partir de la revolución industrial y como consecuencia, se ha incrementado la temperatura global.

Los efectos del cambio climático se han reflejado, en el incremento de la temperatura media anual entre 1880 y 2012 de $0.85^{\circ} \mathrm{C}$; el nivel medio del mar ha aumentado, en promedio, $3.2 \mathrm{~mm}$./año en las últimas dos décadas, si se considera el escenario de mayores emisiones de gases de efecto invernadero, al año 2100 , la temperatura global podría elevarse hasta $4^{\circ} \mathrm{C}$ y un incremento en el nivel del mar de casi un metro con respecto a lo observado entre 1980 y 1999. a fines del S. XXI (Organización de las Naciones Unidas, 2017). 
Para estudiar las posibles consecuencias de la alteración de la composición de la atmósfera, el Programa de Naciones Unidas para el Medio Ambiente (PNUMA) en conjunto con la Organización Meteorológica Mundial (OMM), fue constituido el IPCC que a la fecha ha publicado cinco reportes cuyo objetivo es reunir el conocimiento científico, socioeconómico y técnico sobre el cambio climático (IPCC, 2015)

En México, el Instituto Nacional de Ecología y Cambio Climático (INECC) es el órgano gubernamental creado a partir de la Ley General de Cambio Climático para coordinar y llevar a cabo proyectos de investigación científica y tecnológica en conjunto con instituciones académicas públicas y privadas. A partir de ello surge el proyecto Actualización de escenarios de cambio climático para estudios de impactos, vulnerabilidad y adaptación en México y Centroamérica, financiado por el Fondo de Medio Ambiente Mundial (FMAM) y administrado por el Programa de Naciones Unidas para el Desarrollo (PNUD). (Cavazos, Salinas et al., 2013; Quinta Comunicación Nacional, 2012 en Fernández et al., 2015).

Dicho proyecto incluye 15 modelos generales de circulación atmosférica elaborados por instituciones de diversas partes del mundo e integran a la Quinta Comunicación Nacional y que a su vez fueron utilizados para la elaboración del modelo Reliability Ensemble Averaging, REA, así mismo, se proyectaron datos de temperatura y precipitación a tres horizontes temporales: cercano (2015-2039); intermedio (2045-2069) y lejano (20752099), además se generó un sistema interactivo de consulta y descarga de bases de datos de código abierto. (Fernández et al. 2015; INECC, 2016).

El modelo $R E A$ presenta dos trayectorias representativas de concentraciones o forzamientos radiativos; la 4.5 (bajas emisiones) y la 8.5 (altas emisiones) y una resolución espacial de 30"x30" (Fernández et al., 2015).

\section{EI bosque de oyamel}

El ecosistema del bosque de oyamel brinda refugio a gran cantidad de biodiversidad, en él se han logrado identificar hasta 510 especies de plantas, sin embargo, solo el $8 \%$ de ellas se encuentran de manera común en estas áreas (Sánchez et al., 2005).

El oyamel, además, sirve como sumidero de $\mathrm{CO}_{2}$, de acuerdo con la Procuraduría de Ordenamiento Territorial (2010) el promedio de almacenamiento de carbono registrado en el suelo de conservación de la Ciudad 
de México es de 93.4 ton/ha, en tanto que, Razo et al. (2015) encontraron que los bosques conservados presentaron una menor capacidad de secuestro de carbono (62.6 ton/ha.) en contraste, los bosques alterados con árboles jóvenes alcanzan hasta 138.6 ton/ha. en el PN El Chico, Hidalgo.

El hábitat del oyamel se encuentra restringido y disperso debido principalmente a que requiere de condiciones específicas de temperatura y humedad, se le considera como un bosque relicto de periodos glaciales (Rzedowski \& McVaugh en Guerrero et al., 2014).

Los bosques de Abies religiosa se localizan en altitudes superiores a los 2400 hasta los $3600 \mathrm{msnm}$ o incluso $3800 \mathrm{msnm}$, en laderas protegidas de la radiación solar y de los fuertes vientos, así mismo, donde la humedad es alta y la lluvia apreciable se presenta en al menos 100 días a lo largo del año (Challenger 1998; Rzedowski, 2006; Villers et al., 2006) (figura 1).

Díaz et al. (2011) indican los requerimientos ambientales del bosque de oyamel en el cual consideran cinco variables distintas y establecen cuatro categorías de acuerdo con lo favorable de las condiciones (tabla 1).

Tabla 1. Requerimientos ambientales del oyamel.

\begin{tabular}{|c|c|c|c|c|c|}
\hline \multirow{3}{*}{ Especie } & Variable & \multicolumn{4}{|c|}{ Categoría } \\
\cline { 3 - 6 } & Adecuada & $\begin{array}{c}\text { Moderadamente } \\
\text { adecuada }\end{array}$ & $\begin{array}{c}\text { Poco } \\
\text { adecuada }\end{array}$ & $\begin{array}{c}\text { No } \\
\text { adecuada }\end{array}$ \\
\hline $\begin{array}{c}\text { Abies } \\
\text { religiosa }\end{array}$ & $\begin{array}{c}\text { Precipitación anual } \\
(\mathrm{mm})\end{array}$ & $1000-1800$ & $800-1000$ & $700-800$ & $<700$ \\
& & $>1800$ & & \\
& $\begin{array}{c}\text { Temperatura media } \\
\text { anual }\left({ }^{\circ} \mathrm{C}\right)\end{array}$ & $8-14$ & $7-8$ & $6-7$ & $<6$ \\
& & $14-16$ & $16-20$ & $>20$ \\
& $\begin{array}{c}\text { Temperatura del mes } \\
\text { más frío }\left({ }^{\circ} \mathrm{C}\right)\end{array}$ & $6-10$ & $4-6$ & $3-4$ & $<3$ \\
& & $10-12$ & $12-16$ & $>16$ \\
& Temperatura del mes & $10-14$ & $8-10$ & $7-8$ & $<7$ \\
& más cálido $\left({ }^{\circ} \mathrm{C}\right)$ & & $14-18$ & $18-22$ & $>22$ \\
& Periodo seco (meses) & $0-3$ & 4 & $5-6$ & $>7$ \\
\hline
\end{tabular}

Fuente: Díaz et al. (2011).

\section{Delimitación del área de estudio}

El área de estudio se delimita al Norte con el Parque Nacional El Chico (20 13 ' 25'N); al Sur Parque Nacional Izta-Popo (18 59'00”N); al Este el lago de Cuitzeo (10059'10") y al Oeste el Parque Nacional Pico de Orizaba (97¹2’00’) (figura 1). 


\section{Metodología}

La línea base de las condiciones climáticas se construyó a partir de los datos mensuales de las estaciones climatológicas de CLICOM del Servicio Meteorológico Nacional. El periodo de tiempo considera una dimensión temporal de 1950 hasta 2010, así como al menos 20 años de datos continuos y $90 \%$ de disponibilidad de los mismos.

La validez de los datos depende principalmente de tres factores; 1) tamaño de la muestra; 2) homogeneidad; 3) coincidencia temporal a fin de contrastarlos (Bello, 2014).

Se utilizó el método estadístico de prueba de homogeneidad por rachas bajo el criterio de Doorembos. Se aplica a los datos de precipitación y consiste en dos etapas: 1) obtención de media y median de la serie; 2) cálculo de desvíos o cambios de signo. Si el número de desvíos se encuentra dentro del rango establecido por Doorembos, la serie se considera homogénea, de lo contrario la estación se descarta (Cruz et al., 2012).

Se calcularon datos de temperatura media, máxima, mínima y precipitación a partir de la determinación del gradiente altitudinal mediante ecuaciones de regresión lineal simple para puntos aleatorios cercanos a la cima del volcán Tláloc, Iztaccíhuatl, Popocatépetl y La Malinche.

El total de estaciones climatológicas, incluyendo los puntos aleatorios calculados fueron 168; Ciudad de México, 20; Estado de México, 56; Morelos, 8; Tlaxcala, 15; Puebla, 21; Hidalgo, 25; Veracruz, 12; Michoacán, 11 (figura 2).

La base de datos quedó integrada por un total de cinco variables climatológicas:

1. Temperatura media anual

2. Temperatura media máxima anual

3. Temperatura media mínima anual

4. Precipitación media anual

5. Unidades fototérmicas anuales 
Figura. 1. Ubicación de estaciones climatológicas y bosque de oyamel.

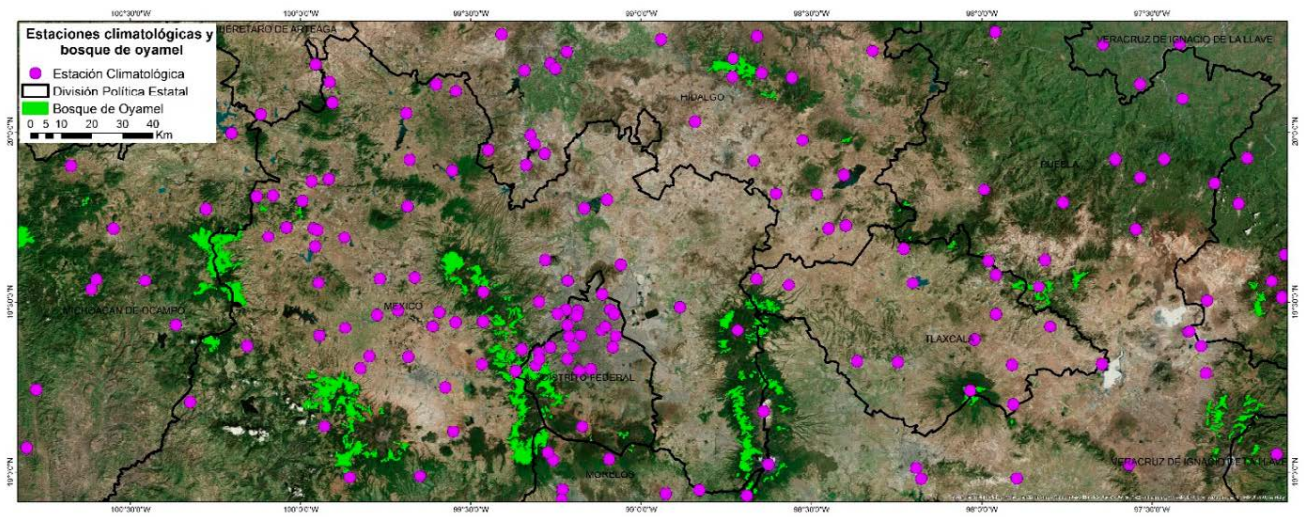

Fuente: CLICOM, INEGI

Se trabajó con el modelo de circulación regional mexicano $R E A$ con horizonte lejano (2075-2099) con los forzamientos radiativos disponibles (4.5 y 8.5) y resolución de 30" x 30" por píxel. Los datos mensuales de las variables climatológicas se integraron a un entorno SIG, de tal manera que se obtuvieron los datos anualizados correspondientes a las 168 estaciones.

Además, se seleccionaron tres de las estaciones ubicadas en inmediaciones del bosque. Se analizó el comportamiento de las condiciones de temperatura y precipitación a lo largo del año para así contrastar las normales climatológicas con las proyecciones del modelo REA mediante climogramas de Ball.

Para la distribución potencial se analizó mediante el software MaxEnt 3.3.3. el cual basa su funcionamiento en el principio de máxima entropía, que consiste en la identificación de las relaciones existentes entre la localización real de las especies y las características biofísicas y ambientales, de tal manera que es posible estimar la probabilidad de la distribución y cuya ecuación básica se expresa del siguiente modo:

$$
H(\hat{\pi})=-\sum_{x \in X} \hat{\pi}(x) \ln \hat{\pi}(x)
$$

Donde $\pi$ es la distribución probable desconocida, $\mathrm{x}$ como el conjunto de píxeles en el área de estudio. La distribución $\pi$ asigna una probabilidad 
no negativa $\pi(\mathrm{x})$ para cada píxel $\mathrm{x}$ sumando $1 . \pi$ también se define como una distribución probable la cual queda definida como $\widehat{\pi}$; $\ln$ indica logaritmo natural. La entropía es no negativa y es como máximo, el logaritmo natural de los elementos contenidos en X (Kumar \& Stohlgren, 2009; Martínez, 2010; Phillips et al., 2006).

Las tablas de puntos georreferenciados se obtuvieron a partir de la capa vectorial de Uso de Suelo y Vegetación Serie V escala 1: 250000 de INEGI y por el otro, archivos ráster en formato ASCII los cuales contienen todas las variables climatológicas a trabajar.

Estos archivos ráster son el resultado de la interpolación de las variables con la ayuda de Sistemas de Información Geográfica (SIG) ya que esta herramienta permite trasladar al espacio geográfico los datos aportados por las estaciones.

El método de interpolación trabajado fue Kriging simple con superficie predictiva y distribución base de t-student, esto debido a que presentó los mejores niveles de desviación estándar a comparación de otros métodos analizados en cada una de las variables (tabla 2).

Tabla 2. Desviación estándar de variables climatológicas con Kriging simple

\begin{tabular}{|c|c|}
\hline Variable & Desviación Estándar \\
\hline Temperatura Media & 3.9 \\
Temperatura Máxima & 4.1 \\
Temperatura Mínima & 3.4 \\
Precipitación & 504.2 \\
Unidades fototérmicas & 176.4 \\
Temperatura Mínima forzamiento 8.5 & 2.9 \\
Temperatura Máxima forzamiento 8.5 & 3.2 \\
Temperatura Media forzamiento 8.5 & 2.8 \\
Precipitación forzamiento 8.5 & 423.9 \\
Unidades fototérmicas forzamiento 8.5 & 143.7 \\
Temperatura Mínima forzamiento 4.5 & 2.8 \\
Temperatura Máxima forzamiento 4.5 & 3.3 \\
Temperatura Media forzamiento 4.5 & 2.8 \\
Precipitación forzamiento 4.5 & 481.4 \\
Unidades fototérmicas 4.5 & 130.1 \\
\hline
\end{tabular}

Fuente: Elaboración propia. 
Los productos obtenidos por el procesamiento incluyen entre otros, archivos de formato ASCII cuyos valores van de 0 a 1 , donde los valores cercanos a cero representan la menor probabilidad de distribución, mientras que los valores cercanos a 1 son aquellos cuya potencialidad es mayor. El formato de salida es compatible en el ambiente SIG, con lo que es posible realizar el análisis espacial de los datos.

La categorización de los valores de cada escenario se realizó tomando como referencia de la propuesta por Díaz et al. (2011) a la que se añadió una quinta; a) muy adecuado; b) adecuado; c) moderadamente adecuado; d) poco adecuado; e) no adecuado.

\section{Resultados \\ Análisis puntual de estaciones climatológicas}

Figura. 2. Climogramas de estaciones climatológicas. A) Desierto de los Leones. B) Presa Iturbide. C) San Antonio Arroyo Prieto.
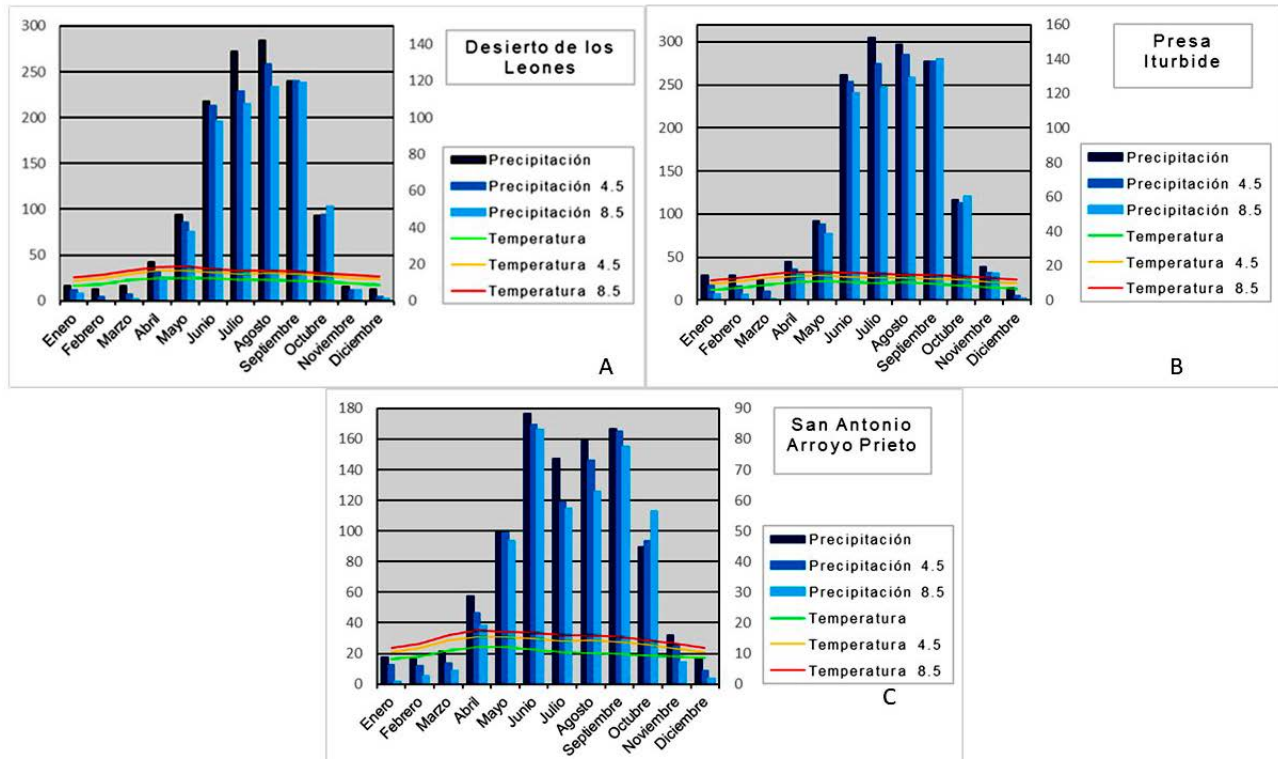

Fuente: Elaboración propia.

La estación climatológica "Desierto de los Leones" (Álvaro Obregón, Ciudad de México), se encuentra a $2995 \mathrm{msnm}$, reporta un periodo de 
funcionamiento de 26.7 años (1961-1987) y un porcentaje de datos total del $99 \%$ (Figura $2(\mathrm{~A})$ ).

La temperatura media anual de la línea base es de $10.6{ }^{\circ} \mathrm{C}$, mayo representa la temperatura más alta del año $\left(12.5^{\circ} \mathrm{C}\right)$ en contraste con enero que es el mes más frío $\left(8.2^{\circ} \mathrm{C}\right)$; ello significa una amplitud térmica de $4.3{ }^{\circ} \mathrm{C}$.

La estación lluviosa se establece a partir del mes de abril y se extiende hasta octubre. La precipitación acumulada anual es de $1316.9 \mathrm{~mm}$ y las lluvias más copiosas ocurren durante el mes de agosto $(284.1 \mathrm{~mm})$ y el de menor cantidad es el de diciembre $(12.4 \mathrm{~mm})$; la temporada de estiaje cubre un periodo de 4 meses.

En el escenario propuesto REA 4.5 la temperatura media anual muestra un aumento de $3.4{ }^{\circ} \mathrm{C}$ respecto al promedio; mayo se mantiene como el mes más cálido del año con $16.5^{\circ} \mathrm{C}$, en contraste, el más frío es enero con $11.1^{\circ} \mathrm{C}$ lo que representa un incremento de $4{ }^{\circ} \mathrm{C}$ y $2.9^{\circ} \mathrm{C}$ respectivamente; con una amplitud térmica de $5.5^{\circ} \mathrm{C}$ a lo largo del año

En cuanto a la precipitación anual muestra una disminución de $9.5 \%$ al ubicarse en $1191.2 \mathrm{~mm}$. El máximo de lluvias se presenta durante agosto con una disminución de del $9 \%$ con respecto a la línea base; el mes más seco se traslada a febrero, al reducirse un $60 \%$ en relación con la normal actual. La temporada seca del año muestra un alargamiento que pasa de cuatro meses en la actualidad a seis meses. En tanto que la extensión de la estación lluviosa se mantiene desde mayo hasta octubre.

El escenario REA 8.5 exhibe condiciones más extremas en general; la temperatura media anual se ubica en $\operatorname{los} 15.7^{\circ} \mathrm{C}$, es decir, $4.9^{\circ} \mathrm{C}$ arriba del escenario base; mayo alcanza los $18.6{ }^{\circ} \mathrm{C}$ lo que representa $6.1{ }^{\circ} \mathrm{C}$ por arriba del mismo mes en la actualidad. Por su parte, el mes más frío se mantiene en enero con $12.6{ }^{\circ} \mathrm{C}, 4.4{ }^{\circ} \mathrm{C}$ más. La amplitud térmica alcanza los $6^{\circ} \mathrm{C}$.

La lluvia acumulada anual exhibe una reducción de 15.6\% al ubicarse en $1191.2 \mathrm{~mm}$; el mes más lluvioso se traslada al mes de septiembre donde la disminución es del $1 \%$ con $237.6 \mathrm{~mm}$, en contraste con el mes de agosto que muestra una disminución de $17.9 \%$; el mes más seco es febrero donde la precipitación se reduce al $100 \%$. La sequía se presenta durante seis meses a lo largo del año y la temporada de lluvias se mantiene desde mayo hasta octubre. 
La segunda estación utilizada para el análisis puntual es "Presa Iturbide" (Isidro Fabela, Estado de México) se ubica a $3290 \mathrm{msnm}$, posee 33 años de funcionamiento (1977-2010) y un porcentaje de datos del $95.1 \%$ (Figura 2 (B)).

La estación reporta una temperatura media anual de $8.9^{\circ} \mathrm{C}$, la temperatura máxima se presenta durante mayo con un valor de $10.9^{\circ} \mathrm{C}$, mientras que la temperatura mínima se observa en enero alcanzando $6.3^{\circ} \mathrm{C}$. La amplitud térmica se observa de $4.6^{\circ} \mathrm{C}$.

La lluvia acumulada anual se ubica en los $1529.2 \mathrm{~mm}$ y la mayor precipitación se registra en durante julio con $304.8 \mathrm{~mm}$, sin embargo, el más seco es diciembre con $14.2 \mathrm{~mm}$. La mayor parte del año puede considerarse dada la relación temperatura-precipitación como húmedo, exceptuando diciembre, aun así, es posible distinguir un incremento significativo en las precipitaciones durante mayo, en lo que puede considerarse el establecimiento de la temporada de lluvias la cual se extiende hasta octubre, momento en el que se percibe el importante descenso de las mismas.

El escenario REA 4.5 da como resultado el incremento de la temperatura media anual hasta $12.2{ }^{\circ} \mathrm{C}$, un incremento de $3.3{ }^{\circ} \mathrm{C}$; el mes más cálido se presenta en mayo con $14.1{ }^{\circ} \mathrm{C}, 3.2^{\circ} \mathrm{C}$ más; la temperatura mínima se aprecia durante enero con un valor de $9.6{ }^{\circ} \mathrm{C}, 3.3{ }^{\circ} \mathrm{C}$ por arriba de la cifra actual; la magnitud de la amplitud térmica es de $4.5^{\circ} \mathrm{C}$.

La lluvia acumulada presenta un descenso en términos anuales de $7.9 \%$ con respecto a la línea base, con $1408.1 \mathrm{~mm}$; agosto, por su parte, se coloca con la mayor cantidad de precipitación con $285.1 \mathrm{~mm}$; julio que en la línea base es la mayor cantidad de precipitación disminuye $10 \%$ hasta $274.3 \mathrm{~mm}$; en tanto, el mes con la menor cantidad de lluvia es diciembre con $5.1 \mathrm{~mm}, 65 \%$ menos.

En el escenario $R E A 8.5$ la temperatura media anual es de $14.4{ }^{\circ} \mathrm{C}$, $5.5^{\circ} \mathrm{C}$ más que en la línea base; la temperatura máxima se presenta durante abril y mayo con $16.7{ }^{\circ} \mathrm{C}$, por su parte, la mínima se observa en enero con $11.6^{\circ} \mathrm{C}$, incrementos de hasta $6.2^{\circ} \mathrm{C}$ y $5.3{ }^{\circ} \mathrm{C}$ respectivamente. Ello representa que la amplitud térmica se ubica en $5.1^{\circ} \mathrm{C}$.

La precipitación advierte un porcentaje de disminución de $14.6 \%$ en el acumulado anual; el mes que concentra la mayor cantidad de lluvia se desplaza de julio, donde se ubica en la línea base, a septiembre donde se observa incluso un ligero incremento con respecto al valor inicial $(0.9 \%)$, 
en contraste con julio que se redujo $18.9 \%$. Diciembre se presenta como el más seco en donde se observa una reducción de $81.5 \%$ con respecto a la normal actual; los meses secos sumen un total de cinco, mayo marca el inicio de la temporada húmeda que se extiende hasta octubre. Destacan los meses de septiembre y octubre en los que se advierten leves incrementos en los acumulados de lluvia.

En tanto la estación "San Antonio Arroyo Prieto" (Ixtacamaxtitlán, Puebla), se localiza a $3065 \mathrm{msnm}$ tiene un periodo de funcionamiento 54.9 años (1954-2009) y concentra el 92.4\% de los datos (figura 2(C)).

La temperatura media anual actual registra $10{ }^{\circ} \mathrm{C}$; abril y mayo son los meses donde se presenta la temperatura más alta con $12.1^{\circ} \mathrm{C}$ mientras que la mínima se encuentra en enero con $8.1^{\circ} \mathrm{C}$, de tal manera que la amplitud térmica es de $4{ }^{\circ} \mathrm{C}$.

La precipitación de la estación registrada fue de $998.4 \mathrm{~mm}$ anuales, en donde el mes más lluvioso es junio con $176.4 \mathrm{~mm}$ y el menos lluvioso es febrero con $16.3 \mathrm{~mm}$. En total se observan 10 meses húmedos por dos secos; las altas precipitaciones comienzan a observarse a partir de mayo hasta octubre. Además, se presenta canícula durante en el mes de julio para alcanzar el segundo máximo en septiembre.

Las condiciones en el modelo REA 4.5 presentan una temperatura media anual de $13.2{ }^{\circ} \mathrm{C}, 3.2{ }^{\circ} \mathrm{C}$ más en comparación a la base actual; la temperatura máxima se advierte durante abril, con $15.5^{\circ} \mathrm{C}$, lo que significa una diferencia de $3.4^{\circ} \mathrm{C}$ a la normal existente; la temperatura mínima ocurre en enero con $10.3{ }^{\circ} \mathrm{C}$, es decir, un aumento de $2.2^{\circ} \mathrm{C}$. La amplitud térmica se ubica en $5.2^{\circ} \mathrm{C}$.

La lluvia experimenta un decrecimiento de $8.8 \%$ al alcanzar la cantidad de $910 \mathrm{~mm}$ anuales; el mes más lluvioso se presenta en junio, donde la variación es de $3.9 \%$ negativo, equivalente a $169.4 \mathrm{~mm}$; en contraste el más seco es diciembre donde se presenta una disminución de $50 \%$ de la precipitación al quedar en $8.4 \mathrm{~mm}$. El periodo de aridez se extiende a lo largo de cuatro meses por ocho de humedad; la temporada de lluvias copiosas abarcan de mayo hasta octubre, este último mes muestra un incremento de $3.9 \%$ para alcanzar $93.2 \mathrm{~mm}$. Se presenta un periodo de sequía intra estival durante julio, pero más acusada que en la actualidad mientras que el segundo punto máximo de lluvias se mantiene en septiembre. 
En el modelo REA 8.5 establece una temperatura media anual de $14.9^{\circ} \mathrm{C}$, lo que representa una diferencia de $4.9^{\circ} \mathrm{C}$ con respecto a la normal actual; la temperatura máxima se ubica durante mayo con $17.2^{\circ} \mathrm{C}, 5.1$ ${ }^{\circ} \mathrm{C}$ por arriba; la temperatura mínima se presenta en enero con $11.6^{\circ} \mathrm{C}$ lo que significa un incremento de $3.5^{\circ} \mathrm{C}$. La amplitud térmica es de $5.6^{\circ} \mathrm{C}$.

El caso de la precipitación tiene una variación negativa de $16.1 \%$ con una cantidad de 837. mm de lluvia anual; la mayor cantidad de lluvia se presenta durante junio, sin embargo, tiene una disminución de $6 \%$ al presente; por su parte, el más seco es enero con solo $1.2 \mathrm{~mm}$, es decir, disminuye $93.2 \%$. En este escenario se observan un total de cinco meses de aridez, por siete húmedos; el establecimiento de la temporada de lluvias se aprecia desde mayo hasta octubre, este último presenta también un incremento en la cantidad acumulada, de $21.2 \%$ para registrar $113 \mathrm{~mm}$. $\mathrm{Al}$ igual que en los otros escenarios, se presenta canícula durante julio, sin embargo, ésta se presenta de manera más acusada que en los anteriores. 
Gabriel Alexis Araiza-Olivares

Efectos del cambio climático en la distribución del bosque de oyamel

\section{Análisis espacial}

Figura. 3. Distribución potencial de oyamel. A) Escenario base; B) Escenario REA 4.5; C) Escenario REA 8.5.
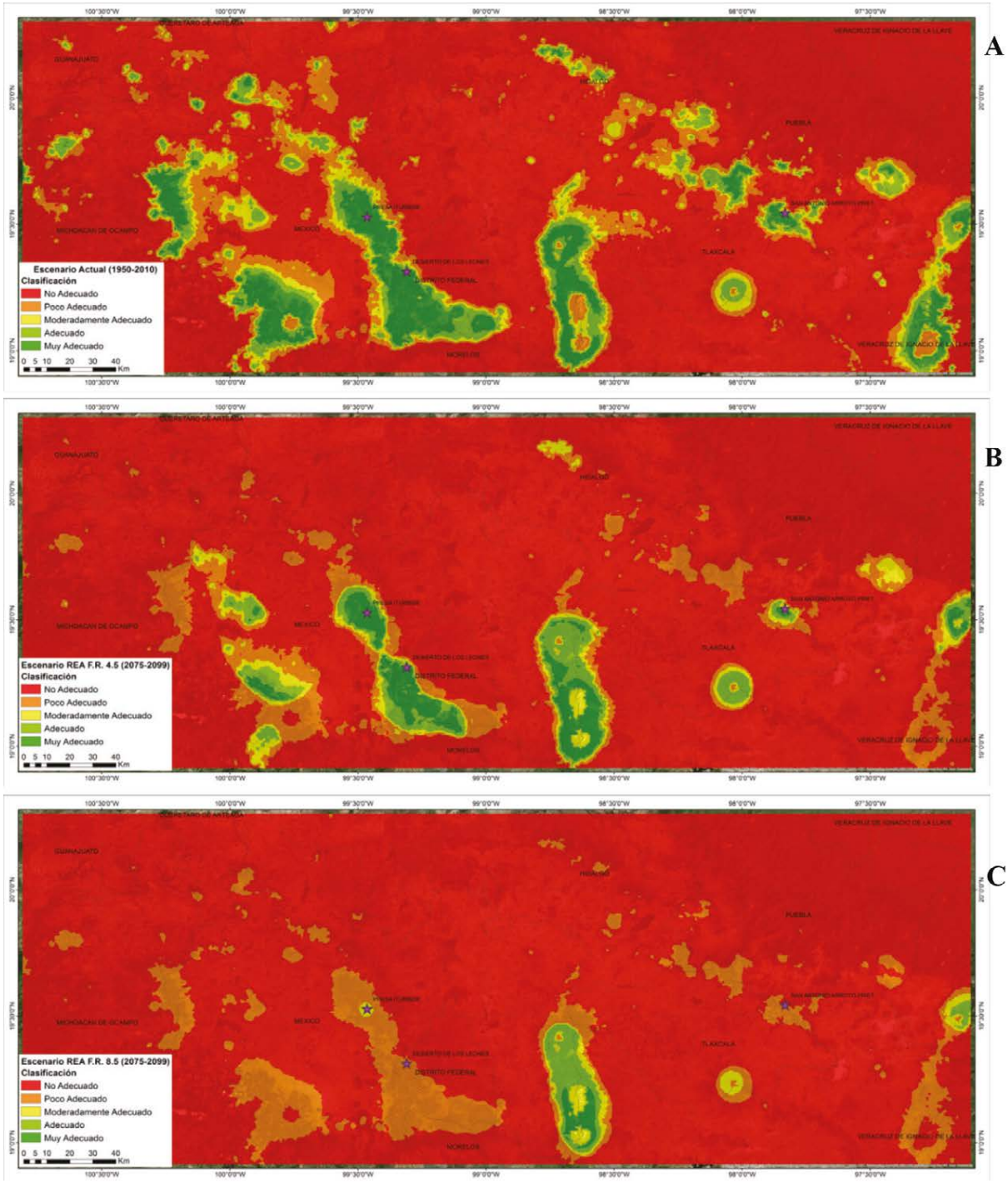

Fuente: Elaboración propia. 
Las condiciones ambientales que se presentan propicias para la distribución potencial bajo las condiciones actuales son amplias, principalmente en las sierras circundantes a la Cuenca de México, Valle de Toluca y Valle de Puebla-Tlaxcala. Las distintas categorías se presentan a manera de anillos concéntrico conforme las condiciones del relieve.

En la línea base (figura 4 (A)) la franja que se clasifican como muy adecuado y adecuado se presentan a partir de los $2800 \mathrm{msnm}$ hasta los 3800 msnm en sitios como la RBB Mariposa Monarca, Nevado de Toluca; en la Sierra de las Cruces y Ajusco-Chichinautzin se ubica alrededor de los $2900 \mathrm{msnm}$ hasta los $3800 \mathrm{msnm}$ aproximadamente; en tanto que en la Sierra Nevada a partir de los $3000 \mathrm{y}$ hasta los $3800 \mathrm{msnm}$; para el volcán La Malinche va de 3000 a 3600 msnm; en el área del Pico de Orizaba y Cofre de Perote desde los 3000 hasta los $3800 \mathrm{msnm}$; en la zona de Mineral del Chico inicia alrededor de 2800 y llega a $3000 \mathrm{msnm}$; en tanto que en los límites de Tlaxcala y Puebla se aprecia a partir de 2800 y por encima de $3200 \mathrm{msnm}$. Fuera de esta franja altitudinal la aptitud de albergar al oyamel disminuye.

El escenario REA 4.5 (figura 4 (B)) muestra una importante reducción en las áreas clasificadas como muy adecuado y adecuado y un incremento sustancial en las clasificadas como poco adecuado.

El área de la RBMM pasa de predominantemente muy adecuado y adecuado a poco adecuado; por su parte se observa la fragmentación del bosque en el Nevado de Toluca, Sierra de las Cruces y Ajusco-Chichinautzin; en la Sierra Nevada destaca el ascenso de la clase moderadamente adecuado a las cercanías de las cimas de los volcanes Iztaccíhuatl y Popocatépetl; en el volcán La Malinche se advierte una cierta condición estable; el Pico de Orizaba pasa a una condición poco adecuada mientras que en el Cofre de Perote se mantiene, sin embargo el corredor que unía a estos se ve deteriorado al clasificarse como poco adecuado; en los límites de los estados de Puebla y Tlaxcala las condiciones pasan a ser poco adecuadas, a pesar de ello se mantiene una porción en adecuado en la zona circundante a la Presa Iturbide; al norte, el Parque Nacional El Chico predominan las condiciones moderadamente adecuadas, en detrimento tanto de la muy adecuada como la adecuada.

En el escenario REA 8.5 (figura 4(C)) las condiciones adecuadas se restringen solo a altitudes superiores a $3000 \mathrm{msnm}$, el otro sitio donde se 
mantienen condiciones adecuadas es en el Cofre de Perote en las laderas a barlovento; mientras que las muy adecuadas de 3400 hasta $3800 \mathrm{msnm}$ pero solo en las laderas del Iztaccíhuatl y Popocatépetl; las moderadamente adecuadas se localizan entorno al cono del volcán La Malinche, debajo de los 3000 metros de la Sierra Nevada y por encima de los 3800 , en el Cofre de Perote en las laderas a sotavento.

El resto de las áreas que anteriormente se encontraban en categorías de moderadamente adecuado, adecuado y muy adecuado pasan a poco adecuado o se integran a la clase de no adecuado.

De las variables climatológicas utilizadas para la construcción del modelo, se encontró que la precipitación fue la que aportó mayor cantidad, en tanto las unidades fototérmicas tuvieron nula aportación al mismo; cabe resaltar que se excluye la componente del relieve por no ser de interés para la investigación. La tabla 3 muestra el porcentaje relativo de aporte al modelo de cada elemento climático.

Tabla 3. Aporte al modelo de las variables climáticas

\begin{tabular}{|c|c|}
\hline Variable & Aportación en \% \\
\hline Precipitación & 41.3 \\
T. mínima & 27.5 \\
T. máxima & 27.5 \\
T. media & 3.4 \\
U. fototérmicas & 0 \\
\hline
\end{tabular}

A partir del análisis espacial se pudo obtener el área aproximada de distribución de cada clase para el oyamel, así como la variación de la superficie con respecto a la línea base (tabla 4). 
Tabla 4. Superficie calculada de distribución del bosque de oyamel y su variación

\begin{tabular}{|c|c|c|c|c|c|}
\hline Categoría & $\begin{array}{c}\text { Superficie } \\
\text { Actual (ha.) }\end{array}$ & $\begin{array}{c}\text { Superficie } \\
\text { FR4.5 (ha.) }\end{array}$ & $\begin{array}{c}\text { Superficie } \\
\text { FR8.5 (ha.) }\end{array}$ & $\begin{array}{c}\text { Variación \%o } \\
\text { (FR4.5) }\end{array}$ & $\begin{array}{c}\text { Variación \% } \\
\text { (FR8.5) }\end{array}$ \\
\hline No Propicio & 4840880.7 & 5521716.7 & 5876454.5 & 14.1 & 21.4 \\
Poco propicio & 681950.6 & 462522.5 & 382777.2 & -32.2 & -43.9 \\
$\begin{array}{c}\text { Moderadamente } \\
\text { Propicio }\end{array}$ & 319152.5 & 130850.8 & 40044.1 & -59.0 & -87.5 \\
Propicio & 465609.4 & 225516.1 & 67912.0 & -51.6 & -85.4 \\
Muy Propicio & 78973.5 & 46046.4 & 19464.7 & -41.7 & -75.4 \\
\hline
\end{tabular}

Fuente: Elaboración propia

\section{Conclusiones}

Los bosques de oyamel son importantes ecosistemas que sirven de refugio de cientos de especies de animales, plantas y hongos, algunas de ellas tan representativas como la mariposa monarca; es de los biomas con mayor capacidad de captura de carbono, almacena e infiltra grandes cantidades de agua; provee diversas materias primas como madera usada en ebanistería, leña y resinas de uso industrial, así como ornamental como árboles de navidad.

La presión directa de las actividades humanas como la extracción de la madera de forma clandestina con fines comerciales, el cambio de uso de suelo para abrir nuevos terrenos agrícolas son dos de los principales motivos por los que la superficie ocupada por el oyamel se ha visto reducida, a esto se le suma la del cambio climático.

Las actuales condiciones climáticas lo colocan como sitios con características de alta humedad, escasos periodos de estiaje y con oscilaciones térmicas bajas a lo largo del año.

En términos generales, tanto el REA 4.5 y 8.5 presentan aumentos significativos de la temperatura y la disminución de las precipitaciones acumuladas. Se observa que la amplitud térmica tiende a hacerse más acusada, en tanto que, el periodo de estiaje muestra signos de volverse más aguda.

El corrimiento de la estación lluviosa, así como de la distribución de la cantidad de lluvia puede generar la alteración del ciclo fenológico del oyamel. La sequía intra estival presenta valles más bajos que en la actualidad, lo que generaría estrés adicional sobre el ecosistema. 
Solo el modelo con forzamiento radiativo 8.5 muestra incrementos en la precipitación, coincidente en el mes de octubre para las tres estaciones analizadas.

Las variaciones en temperatura y precipitación como factores tensionantes representarían el aumento de la vulnerabilidad del oyamel al ataque de plagas y enfermedades, así como el potencial de incremento de ocurrencia de incendios forestales. Los modelados de ambas proyecciones muestran la tendencia de migración en la vertical, sin embargo, en altitudes que resulten insuficientes para cubrir sus necesidades puede darse el caso del eventual desplazamiento por otras especies mejor adaptadas.

A pesar de que actualmente la especie no se encuentra enlistada bajo la protección de la NOM-059-SEMARNAT-2010, muestra una tendencia generalizada a reducir su población. La progresiva pérdida de cobertura boscosa debido al cambio climático pondría en peligro de extinción no solo al oyamel sino a las especies que conforman el ecosistema que a su vez significaría el colapso de las cadenas tróficas.

Frente a estos escenarios es imperativo buscar alternativas que busquen proteger al bosque de oyamel y con ello al ecosistema completo mediante la elaboración de planes y programas de manejo especialmente dirigidos y que permitan la participación en la gestión por parte de las comunidades forestales, en su mayoría de origen indígena, en la protección y aprovechamiento sustentable de los recursos. Actualmente se desarrollan distintos proyectos de conservación, ejemplo de ello es la Universidad Michoacana de San Nicolás Hidalgo cuya línea de investigación se enfoca en la migración asistida, la creación de bancos de germoplasma y la instalación de viveros para la producción de árboles con fines de reforestación.

Es recomendable el fortalecimiento y consolidación de la red de estaciones climatológicas, así como el registro y sistematización de datos, a fin de lograr investigaciones con mayores niveles de confiabilidad, y que sirvan para elaborar estudios detallados con la finalidad de servir como una base sólida en la que se sustenten políticas públicas de planeación y conservación a largo plazo. 


\section{Referencias}

Bello, L. (2014). Escenarios de cambio climático regionalizados para la reserva de la biosfera, Sierra Gorda Guanajuato mediante el modelo LARS-WG (Tesis de Licenciatura) Colegio de Geografía. Facultad de Filosofía y Letras. UNAM. 109 pp.

Cavazos, T., Salinas, J., Martínez, B., Colorado, G., de Grau, P., Prieto, R., Conde, A., Quintanar, A., Santana, J., Romero, R., Maya, M., Rosario, J., Ayala, M., Carrillo, H., Santiesteban, O. y Bravo, M. (2013). Actualización de los escenarios de cambio climático para México como parte de los productos de la quinta comunicación nacional. Informe final del proyecto al INECC. Recuperado: http://escenarios. inecc.gob.mx/index $2 . h t m l$

Challenger, A. (1998). Utilización y conservación de los ecosistemas terrestres de México. Pasado, presente y futuro. Comisión Nacional para el Conocimiento y uso de la Biodiversidad, Instituto de Biología de la UNAM, Agrupación Sierra Madre S.C. México. 847 pp.

Cruz, D., Reyes, M. y Bello, L. (2012) Control de Calidad de Datos. En Gómez, L., Medina, M. (Comp.), Guía de Métodos Estadísticos en Climatología. Pp. 20-24, Colegio de Geografía, Facultad de Filosofía y Letras, UNAM.

Díaz, J., Monterroso, A., Tinoco, J., Toledo, M., Conde, C. y Gay, C. (2011). Assessing current and potential patterns of 16 forest species driven by climate change scenarios in México. Atmósfera, Vol. 24, No. 1, pp. 31-52.

Dirección del Parque Nacional Izta-Popo, Subdirección de Cultura para la Conservación, Departamento de Investigación y Monitoreo (2014). Ficha de identificación Abies religiosa. Recuperado: http://iztapopo.conanp.gob.mx/documentos/fichas_de_especies/Abies_religiosa.pdf

Fernández, A., Zavala, J., Romero, R., Conde, A. y Trejo, R. (2015). Actualización de los escenarios de cambio climático para estudios de impactos, vulnerabilidad, y adaptación en México y Centroamérica. Centro de Ciencias de la Atmósfera, Universidad Nacional Autónoma de México. Instituto Nacional de Ecología y Cambio Climático. Secretaria de Medio Ambiente y Recursos Naturales. Recuperado: http://atlasclimatico.unam.mx/AECC/servmapas 
Guerrero, R., González, J. y Castro, A. (2014). Análisis florístico de un bosque de Abies y el bosque mesófilo de montaña adyacente en Juanacatlán, Mascota, Jalisco, México. Botanical Science, Vol. 92, No.4, pp. 541-562.

Instituto Nacional de Ecología y Cambio Climático (2016). Escenarios de cambio climático. Recuperado: http://www.gob.mx/inecc/ acciones-y-programas/escenarios-de-cambio-climatico-80126

Intergovernmental Panel on Climate Change (2014). Cambio Climático 2014. Informe Síntesis. Contribuciones de los grupos de trabajo I, II y III al Quinto Informe de Evaluación del Grupo Intergubernamental de Expertos sobre el Cambio Climático (Equipo Principal de Redacción, R.K. Pachauri y L.A. Meyers (Eds.)). IPCC, Ginebra, Suiza, $157 \mathrm{pp}$.

Intergovernmental Panel on Climate Change (2015). Ficha informativa del IPCC: Fechas y momentos destacados de la historia del IPCC. Recuperado: https://www.ipcc.ch/site/assets/uploads/2018/04/ FS_timeline_es.pdf

Intergovernmental Panel on Climate Change (S/A). ¿Qué es el IPCC? Recuperado: http://www.ipcc.ch/home_languages_main_spanish.shtml

Kumar, S. y Stohlgren, T. (2009). Maxent modeling for predicting suitable hábitat for threatened and endangered tree Canacomyrica monticola in New Caledonia. Journal of Ecology and Natural Environment, Vol. 1, No. 4, pp. 94-98.

Magaña, V. (2004). El cambio climático global: Comprender el problema. En Martínez, J. y Fernández, A. (Coord.) Cambio climático: una visión desde México (pp. 17-27). Ciudad de México, Instituto Nacional de Ecología, Secretaria de Medio Ambiente y Recursos Naturales. Martínez, N. (2010). Apuntes sobre modelación de nichos ecológicos. Laboratorio de evolución molecular y experimental. Instituto de Ecología, UNAM.

Organización de las Naciones Unidas (2017). Los efectos del Cambio Climático y los Cambios Conexos en los Océanos. Resúmen técnico de la Primera Evaluación Integrada del Medio Marino a Escala Mundial Recuperado: https://www.un.org/regularprocess/sites/ www.un.org.regularprocess/files/17-05753_s-impacts-of-climatechange.pdf 
Phillips, S., Anderson, R., Schapire, R. (2006). Maximum entropy modeling of species geographic distributions. Ecological Modelling, No. 190, pp. 231-259.

Procuraduría Ambiental y Ordenamiento Territorial del DF (2010). Cuantificación del carbono almacenado en la vegetación del suelo de conservación del Distrito Federal. Recuperado: http://centro.paot. org.mx/documentos/paot/estudios/IPA-05-2010_Estudio_Carbono_ Difusion_INIFAP-CGEO.pdf

Razo, R., Gordillo, A., Rodríguez, R., Maycotte, C. y Acevedo, O. (2015). Coeficientes de carbono para arbustos y herbáceas del bosque de oyamel del Parque Nacional El Chico. Revista mexicana de Ciencias Forestales, Vol. 6, No. 31, pp. 58-67.

Rzedowski, J. (2006) Vegetación de México. 1era edición digital, Comisión Nacional para el Conocimiento y Uso de la Biodiversidad, México.

Sánchez, A., López, L. y Granados, D. (2005). Semejanza florística entre los bosques de Abies religiosa (H.B.K.) Cham. \& Schltdl. de la Faja Volcánica Transmexicana. Investigaciones Geográficas, No. 56, pp. 62-76. Instituto de Geografía, UNAM.

Villers, L., Rojas, F. y Tenorio, P. (2006). Guía botánica del Parque Nacional Malinche Tlaxcala-Puebla. Centro de Ciencias de la Atmósfera, Instituto de Biología, UNAM. 194 pp. 